\title{
Randomised controlled trials in severe asthma: selection by phenotype or stereotype
}

\author{
Thomas Brown ${ }^{1,3}$, Thomas Jones (10) ${ }^{1,3}$, Kerry Gove (1) ${ }^{2}$, Clair Barber², \\ Scott Elliott ${ }^{1}$, Anoop Chauhan ${ }^{1}$ and Peter Howarth ${ }^{2}$, on behalf of the Wessex \\ Severe Asthma Cohort (WSAC) team ${ }^{4}$
}

Affiliations: ${ }^{1}$ Queen Alexandra Hospital, Portsmouth Hospitals NHS Trust, Portsmouth, UK. ${ }^{2}$ Clinical and Experimental Sciences, Faculty of Medicine, University of Southampton and NIHR Southampton Biomedical Research Centre, Southampton, UK. ${ }^{3}$ These authors contributed equally. ${ }^{4} \mathrm{~A}$ list of the members of the Wessex Severe Asthma Cohort (WSAC) team can be found at the end of this article.

Correspondence: Thomas Brown, Queen Alexandra Hospital, Portsmouth Hospitals NHS Trust, Southwick Hill Road, Cosham, Portsmouth, P063LY, UK. E-mail: thomas.brownaporthosp.nhs.uk

@ERSpublications

RCTs of biological therapies in severe asthma are poorly generalisable with most patients excluded by outmoded disease concepts despite possessing the targetable trait addressed by the treatment http://ow.ly/iog930md0J3

Cite this article as: Brown $\mathrm{T}$, Jones $\mathrm{T}$, Gove $\mathrm{K}$, et al. Randomised controlled trials in severe asthma: selection by phenotype or stereotype. Eur Respir J 2018; 52: 1801444 [https://doi.org/10.1183/ 13993003.01444-2018].

ABSTRACT Previous publications have highlighted the disparity between research trial populations and those in clinical practice, but it has not been established how this relates to randomised controlled trials (RCTs) of phenotype-targeted biological therapies in severe asthma.

Detailed characterisation data for 342 severe asthma patients within the Wessex Severe Asthma Cohort (WSAC) was compared against comprehensive trial eligibility criteria for published phase IIB and phase III RCTs evaluating biological therapies in severe asthma since 2000.

37 RCTs evaluating 20 biological therapies were identified. Only a median of 9.8\% (range 3.5-17.5\%) of severe asthma patients were found to be eligible for enrolment in the phase III trials. Stipulations for airflow obstruction, bronchodilator reversibility and smoking history excluded significant numbers of patients. A median of $78.9 \%$ (range $73.2-86.6 \%$ ) of patients with severe eosinophilic asthma would have been excluded from participation in the phase III licensing trials of interleukin (IL)-5/IL-5R targeted therapies.

Despite including only well characterised and optimally treated severe asthmatics under specialist care within the WSAC study, the vast majority were excluded from trial participation by criteria designed to re-confirm diagnostic labels rather than by biomarker criteria that predict the characteristic addressed by the treatment. 


\section{Introduction}

Asthma affects over 300 million people worldwide with an estimated total annual cost in the UK approaching $£ 5$ billion $[1,2]$. Whilst the majority of people with asthma can be treated effectively with inhaled corticosteroids (ICS) and bronchodilators, 5-10\% with severe asthma suffer persistent symptoms, frequent exacerbations and an accelerated decline in lung function despite treatment [3]. Severe asthma is significantly more expensive with increased healthcare resource usage and high-cost medications accounting for much of this additional cost [4].

Cohort studies and cluster analyses have advanced our understanding of severe asthma, establishing it as a heterogeneous condition encompassing multiple phenotypes with underlying endotypes [5-9] defined by specific pathobiological pathways that underpin the manifestations of the disease. To address the significant unmet clinical need in severe asthma, there has been a focus since the turn of the millennium on developing biological therapies to target specific components of these inflammatory pathways (predominantly in those with Type-2 inflammation) [10]. These targeted interventions are recognised as an important step towards personalised medicine for patients with severe asthma. However, such treatments are expensive mandating that their use is rationalised by high-quality clinical evidence of efficacy and effectiveness, and the use of biomarkers to stratify patients and determine those most likely to benefit from treatment.

Recent expert commentary has proposed that less emphasis be placed on historical definitions of asthma, highlighting that most randomised controlled trials (RCTs) study populations that are poorly generalisable to clinical practice $[11,12]$, with a focus instead on "treatable traits" to identify clinical trial populations who are most likely to benefit from an intervention. Previous studies have shown that only 3.3-6\% of patients with asthma fulfilled the eligibility criteria for the clinical trials upon which asthma guidelines are based $[13,14]$. However, these studies did not focus on patients with severe asthma under specialist care or biological therapies targeting specific asthma phenotypes and thus it is unclear whether a similar impact on the generalisability of trial data exists.

To investigate this we have therefore aligned the data from a large well characterised cohort of severe asthma patients, the Wessex Severe Asthma Cohort (WSAC), with the clinical trial eligibility criteria of published RCTs assessing biological therapies in severe asthma. Additionally, we have compared the profile of the WSAC with published data from other severe asthma cohorts to evaluate how representative this cohort is of the broader severe asthma population, so that the implications of these findings can be fully appreciated.

\section{Methods}

Study design

The WSAC is an observational, cross-sectional study providing detailed characterisation of severe asthma patients recruited from specialist severe asthma clinics at Portsmouth and Southampton Hospitals between April 2009 and January 2014. Study participants had asthma, confirmed by a specialist in accordance with the British Thoracic Society (BTS)/Scottish Intercollegiate Guidelines Network (SIGN) guidelines 2009, which remained poorly controlled with persisting symptoms and exacerbations despite treatment with high-dose ICS (or maintenance systemic corticosteroids), a long-acting $\beta_{2}$-agonist (LABA) and/or alternative controller medications (equivalent to step 4 or step 5 of the Global Initiative for Asthma (GINA) management guidelines for asthma 2017 [15]), as well as focused management of any co-morbid conditions (full inclusion criteria are included in the supplementary material).

\section{Characterisation protocol}

All participants underwent a detailed characterisation protocol including clinical, physiological and biological assessments (full details are included in the supplementary material). The study was funded by the UK Medical Research Council (MRC)/National Institute for Health Research (NIHR) Patient Research Cohorts Initiative and was conducted in accordance with the International Conference on Harmonisation and Good Clinical Practice standards and the ethical principles outlined in the Declaration of Helsinki. Independent ethics committee approval was obtained (MREC No. 09/H0502/37) and all participants provided written, informed consent prior to participation in the study.

\section{Identification of trials and eligibility analysis}

A systematic search was used to identify all phase IIB and phase III RCTs studying novel treatments in severe asthma between January 2000 and January 2018. Abstracts were reviewed and primary publications sought for relevant RCTs (the reference lists of these publications were also reviewed). Key eligibility criteria were extracted from primary publications, published trial protocols and clinical trial databases where available. Each patient from the WSAC was assessed against the eligibility criteria for each trial to determine the numbers that would have been deemed suitable for enrolment and key criteria which 
excluded patients from trial participation were reviewed. Criteria were divided into diagnostic criteria (e.g. airflow obstruction and reversibility) and biomarker criteria (e.g. peripheral blood eosinophil count), with the latter used to identify a specific disease phenotype. Where relevant data was unavailable it was assumed patients remained eligible, with the exception of studies mandating sputum eosinophilia where failure of sputum production precluded enrolment. In addition, each patient was assessed against the National Institute for Health and Care Excellence (NICE) treatment recommendations for biological therapies currently licenced for use in asthma in the UK. The primary outcome was the proportion of patients eligible for each of the RCTs identified.

Role of the funding source

The UK MRC and NIHR provided joint funding for the study but did not contribute to its design or to collection, analysis or interpretation of the data.

\section{Results}

The WSAC enrolled 342 severe asthmatics, all of whom fulfilled the American Thoracic Society (ATS)/ European Respiratory Society (ERS) 2014 definition of severe asthma. A summary of the characteristics of this group is shown in table 1 and further details are included in the supplementary material. The severe asthma patients within the WSAC are demographically comparable to previous cohorts/registries, as detailed in the supplementary material.

37 RCTs, comprising 23 phase II and 14 phase III trials, assessing 20 novel therapies in over 15000 patients with severe asthma were identified. Of these RCTs, 29 (78\%) assessed treatments targeting the Type-2 "high" inflammatory pathway. The most frequent primary endpoint was a reduction in exacerbation frequency ( $71 \%$ of phase III trials).

Only a median of $9.8 \%$ (range $3.5-17.5 \%$ ) of severe asthma patients within the WSAC would have been eligible for enrolment in the phase III trials of biological therapies in severe asthma. The proportion of patients within the WSAC who would have been suitable for enrolment in each RCT is shown in table 2. Whilst there is an increment in eligibility between phase II and phase III RCTs, overall suitability remains low.

Commonly used eligibility criteria and their impact on trial enrolment are highlighted in table 3 . The requirements for persistent airflow obstruction and/or significant bronchodilator reversibility were key reasons for trial exclusion and when both criteria were required only $33.6 \%$ of severe asthma patients in the WSAC remained eligible. The cumulative effect of multiple eligibility criteria dramatically restricts eligibility for trial participation. However, the use of composite inclusion criteria, allowing bronchodilator reversibility, bronchial hyper-responsiveness and/or measures of variable airflow obstruction, modestly increases median eligibility from $7.6 \%$ (range $2.1-30.7 \%$ ) to $15.8 \%$ (range $4.1-39.5 \%$ ).

In table 2, eligibility is subdivided into asthma and biomarker criteria, which demonstrates that the majority of patients are excluded by non-phenotypic criteria. The percentage of patients with blood eosinophil counts of $\geqslant 300$ cells. $\mu \mathrm{L}^{-1}$ who would have been eligible for enrolment in published phase III trials of IL-5 and IL-5R targeted therapies is illustrated in figure 1. Again this demonstrates that most are excluded by non-phenotypic criteria. A similar effect is seen with an eosinophilic population defined as $\geqslant 2 \%$ or $\geqslant 3 \%$ of sputum eosinophils, or by blood eosinophil counts of $\geqslant 150$ cells. $\mu \mathrm{L}^{-1}$ (supplementary material). The median eligibility for RCTs assessing biological agents targeting Type- 2 asthma (8.8\%, range 2.1-26.9\%) was lower than for non-Type-2 asthma (14\%, range 5.3-39.5\%) and comparative RCTs of novel non-biological therapies for severe asthma (33.9\%, range 20.8-76.9\%).

Within the WSAC, $26 \%$ of severe asthmatics were current smokers or ex-smokers with a smoking history of $\geqslant 10$ pack-years. Of those patients who successfully produced sputum (59 out of $90 ; 62 \%$ ) or had a peripheral blood count ( 81 out of $90 ; 90 \%$ ), $56 \%$ had a sputum eosinophil count of $\geqslant 2 \%$ and/or a peripheral blood eosinophil count of $\geqslant 300$ cells $\mu \mathrm{L}^{-1}$ but were not eligible for enrolment in most trials targeting Type- 2 "high" disease due to their smoking status. The impact of smoking on Type-2 biomarker status in the WSAC is shown in table 4.

That less than $50 \%$ of the severe asthmatics in the WSAC who fulfilled the NICE recommendations for treatment with mepolizumab and reslizumab would have been eligible for inclusion in the phase III trials of these therapies (mepolizumab 45.3\%; reslizumab 33.9\%) is demonstrated in figure 2.

\section{Discussion}

The WSAC was established with the aim of evaluating real-world severe asthma patients and is comparable to the severe asthma populations described in previously published cohorts and registries [7, 16-19], from which cluster analyses have identified the currently recognised asthma phenotypes. Despite including only well characterised and optimally treated severe asthmatics under specialist care, the 
TABLE 1 Wessex Severe Asthma Cohort (WSAC) characteristics summary ( $n=342$ )

\section{Patient demographics}

Age years

Female

$49.4 \pm 13.6$

$\mathrm{BMI} \mathrm{kg} \cdot \mathrm{m}^{-2}$

$\mathrm{BMI}>30 \mathrm{~kg} \cdot \mathrm{m}^{-2}$

$29.7(25.6-35.6)$

48.2

Smoking status

Never smoker

Ex-smoker

Current smoker

Ex-smoker pack-years

Current smoker pack-years

$20(8.4-35)$

\section{Asthma history}

Asthma duration years

ICS dose BDP equivalent $\mu \mathrm{g} \cdot$ day $^{-1}$

LABA

LTRA

LAMA

Theophylline

Maintenance OCS

Prednisolone equivalent dose $\mathrm{mg}$

$15.9 \pm 12$

Asthma control

Rescue OCS courses in the previous year

$\geqslant 1$ hospital admissions in the previous year

ACQ6 score

$2.74 \pm 1.24$

ACQ6 score $>1.5$

Previous intensive care admission for asthma

AQLQ score

\section{Comorbidities}

Gastro-oesophageal reflux disease

48.5

PPI use

Rhinosinusitis

Rhinosinusitis treatment

Nasal polyps

\section{Physiological measures}

Pre-brochodilator FEV $1 \%$ predicted

FEV1 reversibility \% from baseline

Reversibility $\geqslant 12 \% \#$

Atopic status

Atopic

\section{Measures of inflammation}

$\mathrm{FeNO}_{50} \mathrm{ppb}$

$\mathrm{FeNO}_{50} \geqslant 50 \mathrm{ppb}$

Sputum inflammatory phenotype

$\geqslant 3 \%$ eosinophilic

$\geqslant 60 \%$ neutrophilic

Mixed granulocytic

Paucigranulocytic

Blood eosinophil count $\times 10^{9}$ per $L$

\section{ATS/ERS severe asthma criteria 2014 [3]}

GINA step $4 / 5$ treatment

ACQ6 score $>1.5^{+}$

$\geqslant 2$ OCS bursts in the previous year ${ }^{+}$

Data are presented as \%, mean \pm SD or median (interquartile range). BMI: body mass index; ICS: inhaled corticosteroids; BDP: beclometasone dipropionate; LABA: long-acting $\beta_{2}$-agonist; LTRA: leukotriene receptor antagonist; LAMA: long-acting muscarinic antagonist; OCS: oral corticosteroids; ACQ6: asthma control questionnaire 6; AQLQ: asthma quality of life questionnaire; PPI: proton pump inhibitor; FEV1: forced expiratory volume in $1 \mathrm{~s} ; \mathrm{FeNO}_{50}$ : exhaled nitric oxide fraction at $50 \mathrm{~mL} \cdot \mathrm{s}^{-1}$ flow rate; ATS: American Thoracic Society; ERS: European Respiratory Society; GINA: Global Initiative for Asthma. " : reversibility testing was performed 15 min after administration of 2.5 mg nebulised salbutamol; " ": atopic was defined as one or more positive skin prick tests; ${ }^{+}: 100 \%$ of patients in the WSAC meet one or more of the four major "poor control" criteria. 


\begin{tabular}{|c|c|c|c|c|c|c|c|}
\hline \multirow[t]{2}{*}{ Target } & \multirow[t]{2}{*}{ Drug } & \multirow{2}{*}{$\begin{array}{c}\text { First } \\
\text { Author }^{\text {I }}\end{array}$} & \multirow[t]{2}{*}{ Year } & \multirow[t]{2}{*}{ Phase } & \multicolumn{3}{|c|}{ WSAC eligibility $\%$} \\
\hline & & & & & $\begin{array}{c}\text { Biomarker } \\
\text { criteria }\end{array}$ & $\begin{array}{l}\text { Asthma } \\
\text { criteria }\end{array}$ & Overall \\
\hline \multirow[t]{3}{*}{$\lg E$} & Omalizumab & Holgate & 2004 & III & 36.84 & 12.57 & 3.51 \\
\hline & Omalizumab & HUMBERT & 2005 & II & 36.84 & 11.70 & 4.09 \\
\hline & Omalizumab & HANANIA & 2011 & III & 36.84 & 18.42 & 6.14 \\
\hline \multirow[t]{13}{*}{ IL-5 } & Mepolizumab & HALDAR & 2009 & ॥ & 25.15 & 23.10 & 7.89 \\
\hline & Mepolizumab & $\mathrm{N}_{\text {AIR }}$ & 2009 & ॥ & 25.15 & 23.10 & 5.56 \\
\hline & Mepolizumab & PAVORD & 2012 & III & 57.31 & 23.10 & 17.54 \\
\hline & Mepolizumab & BEL & 2014 & III & 69.59 & 8.77 & 4.09 \\
\hline & Mepolizumab & Ortega & 2014 & III & 69.59 & 23.98 & 15.79 \\
\hline & Mepolizumab & CHUPP & 2017 & III & 69.59 & 23.39 & 14.91 \\
\hline & Reslizumab & CAstro & 2011 & II & 25.15 & 22.81 & 4.68 \\
\hline & Reslizumab & CASTRO & 2015 & III & 45.32 & 23.68 & 8.77 \\
\hline & Reslizumab & CASTRO & 2015 & III & 45.32 & 23.68 & 8.77 \\
\hline & Reslizumab & BJERMER & 2016 & III & 45.32 & 29.24 & 10.82 \\
\hline & Reslizumab & Corren & 2016 & III & 100.00 & 15.50 & 15.50 \\
\hline & Benralizumab & BLEEKER & 2016 & III & 100.00 & 11.11 & 11.11 \\
\hline & Benralizumab & FitzGerald & 2016 & III & 100.00 & 11.11 & 11.11 \\
\hline \multirow[t]{6}{*}{ IL-13 } & Tralokinumab & PIPER & 2013 & II & 100.00 & 26.90 & 26.90 \\
\hline & Tralokinumab & BRIGHTLING & 2015 & II & 100.00 & 11.40 & 11.40 \\
\hline & Lebrikizumab & CoRren & 2011 & II & 100.00 & 6.14 & 6.14 \\
\hline & Lebrikizumab & HANANIA & 2016 & III & 100.00 & 7.60 & 7.60 \\
\hline & Lebrikizumab & HANANIA & 2016 & III & 100.00 & 7.60 & 7.60 \\
\hline & GSK679586 & De Boever & 2014 & ॥ & 100.00 & 18.42 & 18.42 \\
\hline \multirow[t]{4}{*}{ IL-4Ro } & AMG317 & CORREN & 2010 & II & 73.68 & 3.80 & 3.51 \\
\hline & Dupilumab & Wenzel & 2013 & II & 36.55 & 2.92 & 2.05 \\
\hline & Dupilumab & WENZEL & 2016 & II & 100.00 & 10.53 & 10.53 \\
\hline & Pitrakinra & SLAger & 2012 & ॥ & 100.00 & 23.39 & 23.39 \\
\hline IL-4/5 & Suplatast & TAMAOKI & 2000 & II & 100.00 & 6.43 & 6.43 \\
\hline DP2 & Fevipiprant & GONEM & 2016 & ॥ & 27.19 & 59.65 & 16.67 \\
\hline IL-2R $\alpha$ & Daclizumab & BUSSE & 2008 & II & 73.68 & 6.73 & 5.26 \\
\hline TSLP & Tezepelumab & Corren & 2017 & ॥ & 100.00 & 6.43 & 6.43 \\
\hline c-Kit/PDGF & Masitinib & HUMBERT & 2009 & $\|$ & 99.12 & 5.56 & 5.26 \\
\hline \multirow[t]{2}{*}{ CXCR2 } & Navarixin & NAIR & 2012 & ॥ & 36.84 & 55.56 & 19.88 \\
\hline & AZD5069 & O'BYRnE & 2016 & II & 71.93 & 37.72 & 25.15 \\
\hline IL17RA & Brodalumab & Busse & 2013 & ॥ & 100.00 & 6.73 & 6.73 \\
\hline \multirow[t]{3}{*}{ TNF- $\alpha$} & Etanercept & MORJARIA & 2008 & ॥ & 100.00 & 39.47 & 39.47 \\
\hline & Etanercept & Holgate & 2011 & II & 100.00 & 8.19 & 8.19 \\
\hline & Golimumab & WENZEL & 2009 & $\|$ & 100.00 & 30.70 & 30.70 \\
\hline \multirow[t]{4}{*}{ Non-biological } & BT & CASTRO & 2010 & III & 100.00 & 26.32 & 26.32 \\
\hline & Azithromycin & BRUSSELLE & 2013 & III & 73.98 & 31.58 & 20.76 \\
\hline & Azithromycin & GIBSON & 2017 & III & 100.00 & 76.90 & 76.90 \\
\hline & TLA & STORRAR & 2017 & III & 73.68 & 53.80 & 41.52 \\
\hline
\end{tabular}

IL: interleukin; DP2: prostaglandin $\mathrm{D}_{2}$ receptor 2; TSLP: thymic stromal lymphopoietin; c-kit: proto-oncogene c-Kit; PDGF: platelet-derived growth factor; CXCR2: C-X-C motif chemokine receptor 2; TNF- $\alpha$ : tumour necrosis factor- $\alpha$; BT: bronchial thermoplasty; TLA: temperature-controlled laminar airflow. " : full details are available in the supplementary material; ": full references are available in the supplementary material.

vast majority of patients in the WSAC (90.2\%; range 82.5-96.5\%) would have been excluded from the landmark phase III trials of biological therapies published to date.

Severe asthma is a heterogeneous condition and biological therapies are only likely to benefit subsets of the population. It would seem reasonable to assume that the majority of patients with poorly-controlled severe eosinophilic asthma (despite high-dose inhaled steroids) should have been eligible for inclusion in RCTs of therapies targeting inflammatory mediators of the Type- 2 asthma pathway. However, a median of only 21.1\% (range 13.4-26.8\%) of patients with severe eosinophilic asthma in the WSAC (as defined by a blood eosinophil count of $\geqslant 300$ cells $\mu \mathrm{L}^{-1}$ ) would have been eligible for the phase III trials of these therapies. 
TABLE 3 The impact of commonly used eligibility criteria on Wessex Severe Asthma Cohort (WSAC) eligibility

\begin{tabular}{|c|c|c|c|c|c|}
\hline Eligibility criteria & Trials $\mathrm{n}$ & Criteria variants $n$ & Specific criteria examples & Trials $\mathrm{n}$ & WSAC eligibility $\%$ \\
\hline Airflow obstruction & & & FEV 1 (pre-bronchodilator) $\geqslant 40 \%$ & 11 & 87.72 \\
\hline \multirow[t]{2}{*}{ Bronchodilator reversibility } & $35^{\#}$ & 6 & $\geqslant 12 \%$ increase in $\mathrm{FEV}_{1}$ & 32 & 43.3 \\
\hline & & & $\geqslant 12 \%$ and $200 \mathrm{~mL}$ increase in $\mathrm{FEV}_{1}$ & 16 & 38.9 \\
\hline \multirow[t]{2}{*}{ Exacerbation frequency } & 18 & 5 & $\geqslant 2$ in the last 12 months & 11 & 74 \\
\hline & & & $\geqslant 2$ OCS or $\geqslant 1$ hospital & 3 & 79.5 \\
\hline$A C Q$ & & & $A C Q 6 \geqslant 1.5$ & 8 & 86.6 \\
\hline \multirow[t]{2}{*}{ Smoking status } & 35 & 7 & Current smokers excluded & 34 & 83.3 \\
\hline & & & $<10$ pack-years & 24 & 79.5 \\
\hline \multirow[t]{2}{*}{ ICS dose (BDP equivalent) } & 35 & 9 & $\geqslant 1000 \mu \mathrm{g} \cdot \mathrm{day}^{-1}$ & 16 & 93.9 \\
\hline & & & $\geqslant 2000 \mu \mathrm{g} \cdot$ day $^{-1}$ & 3 & 69.6 \\
\hline \multirow[t]{2}{*}{ OCS use } & 37 & 5 & No & 11 & 65.8 \\
\hline & & & $\leqslant 10 \mathrm{mg} \cdot$ day $^{-1}$ & 6 & 84.2 \\
\hline
\end{tabular}

FEV1: forced expiratory volume in 1s; OCS: oral corticosteroids; ACQ: asthma control questionnaire; ICS: inhaled corticosteroids; BDP: beclometasone dipropionate; ${ }^{\#}: 14$ trials used a composite criterion allowing bronchodilator reversibility or bronchial hyper-responsiveness and in some cases other measures of variable airflow obstruction (e.g. diurnal peak expiratory flow rate variability); ": one trial used the asthma control test.

There is significant heterogeneity in the eligibility criteria used between trials despite the aim of reconfirming a diagnosis of asthma in a similar target population. For example, whilst 30 of the 37 RCTs required demonstrable airflow obstruction, 12 unique criteria were used to define this. Whilst some studies adopted pragmatic composite eligibility criteria to broaden inclusion, many still required specific evidence

Eligible

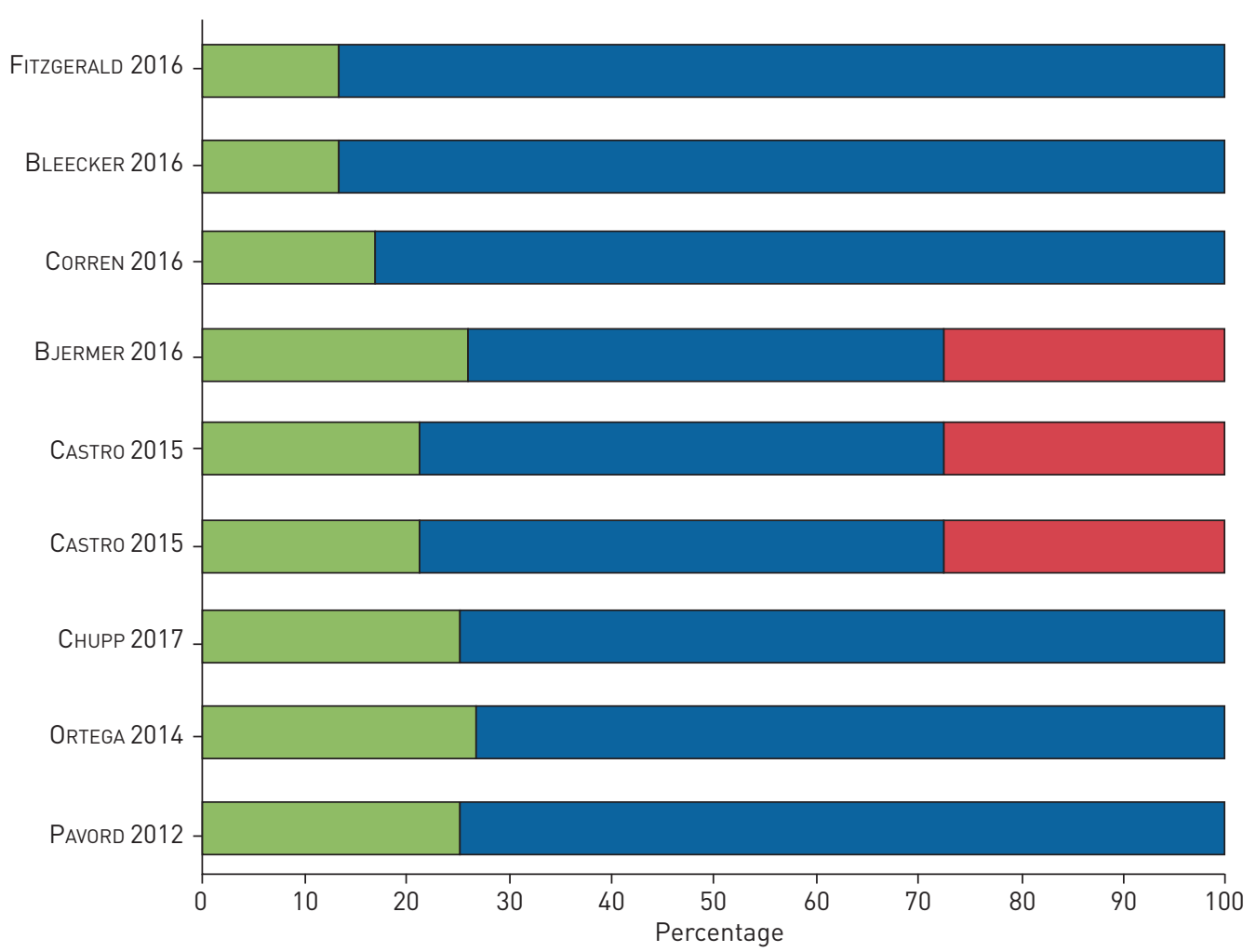

FIGURE 1 Trial eligibility for phase III interleukin (IL)-5 targeted treatments in severe asthmatics with a blood eosinophil count of $\geqslant 300$ cells $\mu \mathrm{L}^{-1}$ 
TABLE 4 The impact of smoking on Type-2 biomarker status in the Wessex Severe Asthma Cohort (WSAC)

\begin{tabular}{|c|c|c|c|c|c|c|c|c|}
\hline \multirow[t]{2}{*}{ Smoking status } & \multirow[t]{2}{*}{ Subjects } & \multirow[t]{2}{*}{ Sputum tests } & \multirow[t]{2}{*}{ Blood tests } & \multicolumn{5}{|c|}{ Biomarker status } \\
\hline & & & & \multicolumn{2}{|c|}{$\begin{array}{c}\text { Sputum } \\
\text { eosinophils }\end{array}$} & \multicolumn{3}{|c|}{ Blood eosinophils } \\
\hline Never smoker & 188 & $107(56.9)$ & $156(83.0)$ & 55 (51.4) & $51(47.7)$ & 98 (62.8) & $79(50.7)$ & 60 (38.5) \\
\hline Ex-smoker $<10$ pack-years & 64 & $43(67.2)$ & 53 (82.8) & 13 (30.2) & 12 (27.9) & $36(67.9)$ & $29(54.7)$ & 19 (35.8) \\
\hline
\end{tabular}

Data are presented as $\mathrm{n}$ or $\mathrm{n}(\%)$.

of bronchodilator reversibility and/or persistent airflow limitation, which dramatically reduced patient eligibility. In the WSAC, of those patients with a sputum eosinophil count of $\geqslant 3 \%$, an asthma control questionnaire (ACQ) score greater than 1.5 and at least one severe exacerbation in the past year, $23 \%$ had no evidence of persistent airflow limitation, $56 \%$ did not have $12 \%$ bronchodilator reversibility and $61 \%$ were excluded when both features were required.

In a similar fashion to other cohorts, $26 \%$ of the severe asthmatics in the WSAC were current smokers $(5.8 \%)$ or ex-smokers with a smoking history of $\geqslant 10$ pack-years $(20.5 \%)$. Whilst it is reported that asthma in smokers is generally associated with non-eosinophilic inflammation [20], a significant proportion in the WSAC did have demonstrable airway eosinophilia. It is recognised that asthmatics who smoke have impaired responsiveness to corticosteroids and suffer more frequent exacerbations and an accelerated rate of lung function decline [21,22]. Arbitrary exclusion of these patients from trials has led to a paucity of evidence upon which to base treatment decisions for them. It is also important to reflect that other factors impacting upon airway biology, including obesity, persisting allergen exposure and bacterial dysbiosis, are not commonly included within trial eligibility criteria, raising a question of equity.

RCTs of Type-2 targeted therapies excluded significantly more participants on the basis of diagnostic criteria than those evaluating non-Type-2 and non-biological therapies. This suggests hyper-selection within this population, further limiting generalisability of the results. Licensing body and healthcare funder recommendations extrapolate from RCTs which we have demonstrated to be poorly representative of real-life severe asthma populations. The disparity between NICE treatment recommendations and the trial populations is highlighted in figure 2. The residual uncertainty this has created as to the benefit of treatment for many patients has led to a reliance on treatment trials. Avoiding eligibility criteria that are

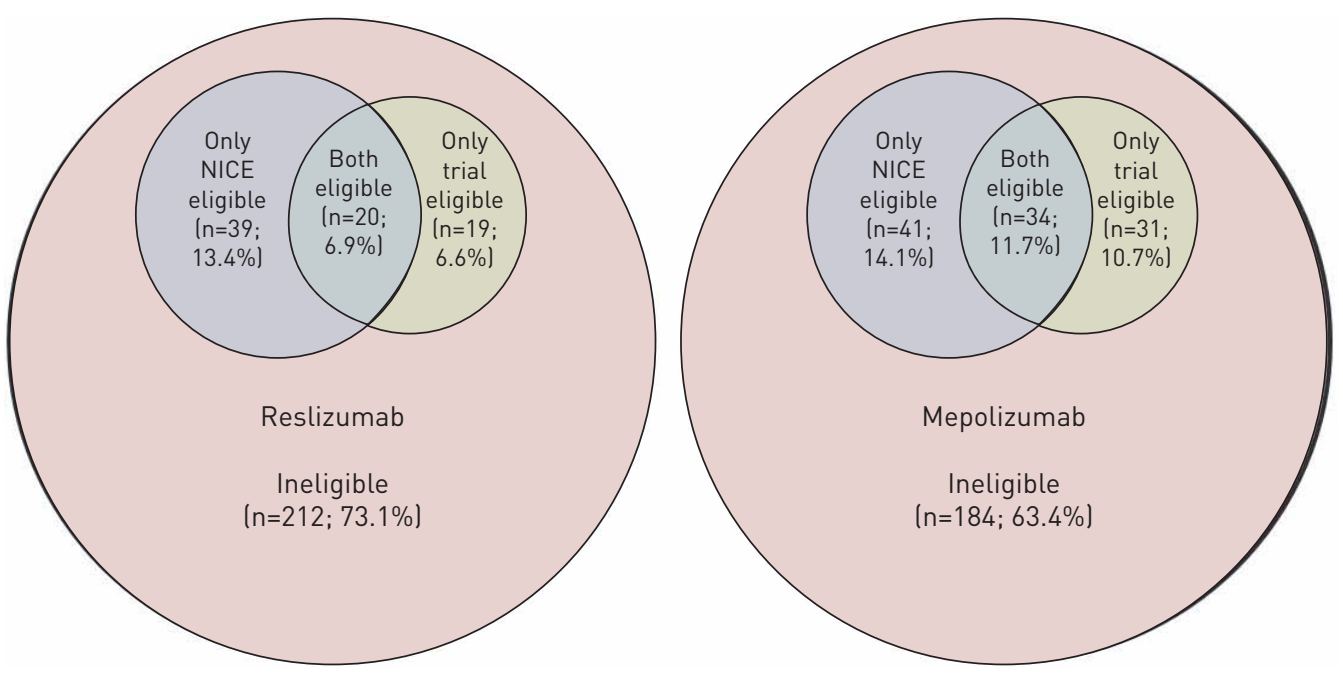

FIGURE 2 A comparison of National Institute for Health and Care Excellence (NICE) treatment recommendations and phase III trial eligibility. All phase III trials were included using eligibility criteria from NICE technology appraisal guidance TA431 (January 2017) and TA479 (October 2017). For full criteria see the supplementary material. 
not relevant to the biological trait targeted would allow for a more inclusive trial population that is better able to be generalised to the subsequent treatment population.

Regulatory authorities have a major influence over the design of RCTs required for product licencing. The European Medicines Agency guidelines for asthma trials specify "the aim should be to study a homogeneous population of patients with asthma" and recommend evidence of reversible airflow obstruction for a secure diagnosis of asthma, substantially limiting trial eligibility [23]. Phase IV pragmatic and non-randomised trials have proved crucial in demonstrating treatment efficacy in those patients excluded from licencing trials [24-26]; however, this creates a significant delay in the generation of evidence for many severe asthma patients and carries the risk of inflating the impact, as real-world evidence is not placebo-controlled. Pragmatic phase III RCTs which better reflect real-world populations and clinical practice may improve external validity and equity of access [27]; however, this will require engagement between clinicians, licencing authorities, funding bodies and the pharmaceutical industry.

Our findings show that RCTs in severe asthma lack external validity, with the majority of patients excluded by criteria designed to confirm arbitrary diagnostic labels rather than by biomarker criteria that predict the characteristic or trait addressed by the treatment [11, 12]. Failure to adopt an exclusively phenotypic approach to trial inclusion will perpetuate the limited generalisability of evidence on effectiveness and health economics used by regulatory bodies. This risks the missing of opportunities for the application of novel therapies and also the propagation of the vast unmet need in severe asthma.

Author contributions: T. Brown, T. Jones, A. Chauhan and P. Howarth contributed to the literatures search, study design, data collection, data analysis, data interpretation and writing of this manuscript. K. Gove, C. Barbar and S. Elliott contributed to the data collection, data analysis and the writing of this manuscript. All other members of the Wessex Severe Asthma Cohort (WSAC) team contributed to the data collection.

Acknowledgements: We would like to thank all of the participants who took part in the Wessex Severe Asthma Cohort (WSAC)

Members of the Wessex Severe Asthma Cohort (WSAC) team: L. Aitkin, S. Babu, P. Dennison, R. Djukanovic, C. Grainge, L. Hewitt, N. Jayasekera, R. Kurukulaaratchy, S. Kerley, L. Lau, D. Laws, J. Owen, E. Ray, D. Reynish, H. Rupani and O. Scullion-Win.

Conflict of interest: T. Brown reports personal fees from Teva, Chiesi Farmaceutici, Novartis and Napp, and nonfinancial support from AstraZeneca, outside the submitted work. T. Jones reports non-financial support from Teva, personal fees and non-financial support from Chiesi Farmaceutici, outside the submitted work. K. Gove has nothing to disclose. C. Barber has nothing to disclose. S. Elliott has nothing to disclose. A. Chauhan reports personal fees and non-financial support from Teva, non-financial support from Boehringer Ingelheim, and personal fees from AstraZeneca, outside the submitted work. P. Howarth reports grants from the Medical Research Council, during the conduct of the study; and is a part-time employee of GlaxoSmithKline.

Support statement: This work was funded by Research Councils UK: Medical Research Council (MRC; G0800649). Funding information for this article has been deposited with the Crossref Funder Registry.

\section{References}

1 Global surveillance, prevention and control of chronic respiratory diseases: a comprehensive approach. Geneva, World Health Organisation, 2007. www.who.int/gard/publications/GARD\%20Book\%202007.pdf

2 Nunes C, Pereira AM, Morais-Almeida M. Asthma costs and social impact. Asthma Res Pract 2017; 3: 1.

3 Chung KF, Wenzel SE, Brozek JL, et al. International ERS/ATS guidelines on definition, evaluation and treatment of severe asthma. Eur Respir J 2014; 43: 343-373.

4 O'Neill S, Sweeney J, Patterson CC, et al. The cost of treating severe refractory asthma in the UK: an economic analysis from the British Thoracic Society Difficult Asthma Registry. Thorax 2015; 70: 376-378.

5 Hinks TSC, Brown T, Lau LCK, et al. Multidimensional endotyping in patients with severe asthma reveals inflammatory heterogeneity in matrix metalloproteinases and chitinase 3-like protein 1. J Allergy Clin Immunol 2016; 138: 61-75.

6 Moore WC, Meyers DA, Wenzel SE, et al. Identification of asthma phenotypes using cluster analysis in the Severe Asthma Research Program. Am J Respir Crit Care Med 2010; 181: 315-323.

7 Shaw DE, Sousa AR, Fowler SJ, et al. Clinical and inflammatory characteristics of the European U-BIOPRED adult severe asthma cohort. Eur Respir J 2015; 46: 1308-1321.

8 Wenzel SE. Asthma: defining of the persistent adult phenotypes. Lancet 2006; 368: 804-813.

9 Lötvall J, Akdis CA, Bacharier LB, et al. Asthma endotypes: a new approach to classification of disease entities within the asthma syndrome. J Allergy Clin Immunol 2011; 127: 355-360.

10 Fajt ML, Wenzel SE. Asthma phenotypes and the use of biologic medications in asthma and allergic disease: the next steps toward personalized care. J Allergy Clin Immunol 2015; 135: 299-310.

11 Pavord ID, Beasley R, Agusti A, et al. After asthma: redefining airways diseases. Lancet 2018; 391: 350-400.

12 Agusti A, Bel E, Thomas M, et al. Treatable traits: toward precision medicine of chronic airway diseases. Eur Respir J 2016; 47: 410-419.

13 Herland K, Akselsen JP, Skjønsberg $\mathrm{OH}$, et al. How representative are clinical study patients with asthma or COPD for a larger "real life" population of patients with obstructive lung disease? Respir Med 2005; 99: 11-19. 
14 Travers J, Marsh S, Williams M, et al. External validity of randomised controlled trials in asthma: To whom do the results of the trials apply? Thorax 2007; 62: 219-233.

15 Global strategy for asthma management and prevention (2017 update). Global Initiative for Asthma 2017. https:// ginasthma.org/wp-content/uploads/2016/01/wms-GINA-2017-main-report-tracked-changes-for-archive.pdf

16 Moore WC, Bleecker ER, Curran-Everett D, et al. Characterization of the severe asthma phenotype by the National Heart, Lung, and Blood Institute's Severe Asthma Research Program. J Allergy Clin Immunol 2007; 119: 405-413.

17 Abraham B, Antó JM, Barreiro E, et al. The ENFUMOSA cross-sectional European multicentre study of the clinical phenotype of chronic severe asthma. Eur Respir J 2003; 22: 470-477.

18 Schleich F, Brusselle G, Louis R, et al. Heterogeneity of phenotypes in severe asthmatics. The Belgian Severe Asthma Registry (BSAR). Respir Med 2014; 108: 1723-1732.

19 Heaney LG, Brightling CE, Menzies-Gow A, et al. Refractory asthma in the UK: cross-sectional findings from a UK multicentre registry. Thorax 2010; 65: 787-794.

20 Thomson NC, Chaudhuri R, Heaney LG, et al. Clinical outcomes and inflammatory biomarkers in current smokers and exsmokers with severe asthma. J Allergy Clin Immunol 2013; 131: 1008-1016.

21 Chaudhuri R, Livingston $\mathrm{E}$, McMahon $\mathrm{AD}$, et al. Cigarette smoking impairs the therapeutic response to oral corticosteroids in chronic asthma. Am J Respir Crit Care Med 2003; 168: 1308-1311.

22 Lange P, Parner J, Vestbo J, et al. A 15-year follow-up study of ventilatory function in adults with asthma. $N$ Engl J Med 1998; 339: 1194-1200.

23 Guideline on the clinical investigation of medicinal products for the treatment of asthma. London, European Medicines Agency, 2015. www.ema.europa.eu/documents/scientific-guideline/guideline-clinical-investigationmedicinal-products-treatment-asthma_en.pdf Date last updated: October 22, 2015

24 Niven RM, Saralaya D, Chaudhuri R, et al. Impact of omalizumab on treatment of severe allergic asthma in UK clinical practice: a UK multicentre observational study (the APEX II study). BMJ Open 2016; 6: e011857.

25 Brusselle G, Michils A, Louis R, et al. "Real-life" effectiveness of omalizumab in patients with severe persistent allergic asthma: the PERSIST study. Respir Med 2009; 103: 1633-1642.

26 Maltby S, Gibson PG, Powell H, et al. Omalizumab treatment response in a population with severe allergic asthma and overlapping COPD. Chest 2017; 151: 78-89.

27 Woodcock A, Vestbo J, Bakerly ND, et al. Effectiveness of fluticasone furoate plus vilanterol on asthma control in clinical practice: an open-label, parallel group, randomised controlled trial. Lancet 2017; 390: 2247-2255. 